International Journal of Pure and Applied Mathematics

Volume 114 No. 2 2017, 191-202

ISSN: 1311-8080 (printed version); ISSN: 1314-3395 (on-line version)

url: http://www.ijpam.eu

doi: 10.12732 /ijpam.v114i2.3

\title{
ON WEAKLY $g(x)$-NIL CLEAN RINGS
}

\author{
Hani A. Khashan ${ }^{1 \S}$, Ali H. Handam² \\ ${ }^{1,2}$ Department of Mathematics \\ Al Al-Bayt University \\ P.O. Box 130095, Al Mafraq, JORDAN
}

\begin{abstract}
Let $R$ be an associative ring with identity and let $g(x)$ be a fixed polynomial in $C(R)[x]$. We define $R$ to be weakly $g(x)$-nil $\left(g(x)\right.$-nil ${ }^{*}$ ) clean if every (non zero) element in $R$ can be written as a sum or a difference of a nilpotent and a root of $g(x)$. For $b \in C(R) \cap U(R)$, we prove that $R$ is weakly (nil) clean if and only if $R$ is weakly $x(x-b)$-(nil) clean. Moreover, for any even integer $k$, we characterize weakly $\left(x^{k}-1\right)$-nil ${ }^{*}$ clean rings as the rings satisfying the nil $k$-involution property. Some other properties are also studied and several examples are given.
\end{abstract}

AMS Subject Classification: 16N40, 16U99

Key Words: nil clean ring, weakly nil clean ring, $\mathrm{g}(\mathrm{x})$-nil clean ring, weakly $\mathrm{g}(\mathrm{x})$-nil clean ring

\section{Introduction}

Let $R$ be an associative ring with identity. Throughout this text, the notations $U(R), J(R), I d(R)$ and $N(R)$ will stand for the set of units, the Jacobson radical, the set of idempotents and the set of nilpotents of $R$, respectively. We denote by $M_{n}(R)$ and $T_{n}(R)$ the rings of all $n \times n$ matrices and all upper triangular matrices over $R$ respectively. Following [15], we define an element $r$ of a ring $R$ to be clean if there is an idempotent $e \in R$ and a unit $u \in R$ such that $r=u+e$. A clean ring is defined to be one in which every element is

Received: $\quad$ September 28, 2016

Revised: $\quad$ March 16, 2017

Published: $\quad$ April 28, 2017

${ }^{\S}$ Correspondence author (c) 2017 Academic Publications, Ltd. url: www.acadpubl.eu 
clean. Similarly a nil clean ring was introduced by Diesel in [11]. An element $r$ in a ring $R$ is said to be nil clean if $r=e+n$ for some idempotent $e \in R$ and a nilpotent element $n \in R$. A ring $R$ is nil clean if each element of $R$ is nil clean. In [4], Breaz, Danchev and Zhou defined a ring $R$ to be weakly nil clean if each element $r \in R$ can be written as $r=n+e$ or $r=n-e$ for $n \in N(R)$ and $e \in \operatorname{Id}(R)$. We refer the reader to $[3,7,8,10,19,4]$ for a survey on nil clean and weakly nil clean rings.

Let $C(R)$ denotes the center of a ring $R$ and $g(x)$ be a polynomial in $C(R)[x]$. Following Camillo and Simón [5], $R$ is called $g(x)$-clean if for each $r \in R$, there exist a unit $u \in R$ and $s \in R$ such that $r=u+s$ and $g(s)=0$. Of course $\left(x^{2}-x\right)$-clean rings are precisely the clean rings.

Nicholson and Zhou [17] proved that if $g(x) \in(x-a)(x-b) C(R)[x]$ where $a, b \in C(R)$ are such that $b$ and $b-a$ are both units in $R$ and ${ }_{R} M$ is a semisimple left $R$-module, then $\operatorname{End}\left({ }_{R} M\right)$ is $g(x)$-clean. Recently, Fan and Yang [12], studied more properties of $g(x)$-clean rings. Among many conclusions, they proved that if $g(x) \in(x-a)(x-b) C(R)[x]$ where $a, b \in C(R)$ are such that $b-a$ is a unit in $R$, then $R$ is a clean ring if and only if $R$ is $(x-a)(x-b)$ clean. Recently, Khashan and Handam [13] have generalized nil clean rings. They defined a ring $R$ to be $g(x)$-nil clean if every element in $R$ is a sum of a nilpotent and a zero of $g(x)$. It is clear that a ring $R$ is $g(x)$-nil clean if and only if for each $r \in R, r=n-s$ where $n$ is a nilpotent and $s$ is a root of $g(x)$. Ashrafi and Ahmadi, [2] have introduced and study weakly $g(x)$-clean rings as rings in which every element is a sum or a difference of a unit and a zero of $g(x)$.

The aim of our paper is to define and study weakly $g(x)$-nil clean rings as a proper generalization of nil clean rings and a proper subclass of weakly $g(x)$-clean rings. For a ring $R$ and $g(x) \in C(R)[x]$, we say that $r \in R$ is weakly $g(x)$-nil clean provided that there exist a nilpotent $n \in R$ and a root $s \in R$ of $g(x)$ such that $r=n+s$ or $r=n-s$. Moreover, $R$ is called weakly $g(x)$-nil clean if every element in $R$ is weakly $g(x)$-nil clean. If every non zero element in $R$ is weakly $g(x)$-nil clean, then $R$ is called weakly $g(x)$-nil* clean.

In the next section we introduce some fundamental facts concerning weakly $g(x)$-nil clean rings and give some necessary examples. In Section 3 , for $b \in$ $C(R) \cap U(R)$, we characterize weakly $x(x-b)$-clean (weakly $x(x-b)$-nil clean) rings in terms of weakly clean (weakly nil clean) rings. Moreover, for any even integer $k$, we characterize weakly $\left(x^{k}-1\right)$-nil* clean rings as the rings satisfying the nil $k$-involution property. 


\section{Weakly $g(x)$-nil Clean Rings}

In this section, we study some of the basic properties of weakly $g(x)$-nil clean rings. Moreover, we give some necessarily examples.

Definition 1. Let $R$ be a ring and $g(x)$ be a fixed polynomial in $C(R)[x]$. An element $r \in R$ is called weakly $g(x)$-nil clean if there exist $n \in N(R)$ and a zero $s$ of $g(x)$ such that $r=n+s$ or $r=n-s$. A ring $R$ is called weakly $g(x)$-nil clean (respectively, weakly $g(x)$-nil* clean) if all of its elements ( respectively, non zero elements) are weakly $g(x)$-nil clean.

Let $g(x) \in C(R)[x], s$ a zero of $g(x)$ and $n \in N(R)$. For $r \in R$, we write $r=n \pm s$ if $r$ is either a sum $n+s$ or a difference $n-s$. Obviously, every $g(x)$-nil clean ring is weakly $g(x)$-nil clean. Since the ring $\mathbb{Z}_{6}$ is a weakly nil clean ring that is not nil clean, then trivially $\mathbb{Z}_{6}$ is a weakly $\left(x^{2}-x\right)$-nil clean ring which is not $\left(x^{2}-x\right)$-nil clean. For a non trivial example, one can easily verify that the ring $\mathbb{Z}_{3}$ is weakly $\left(x^{4}+x\right)$-nil clean but not $\left(x^{4}+x\right)$-nil clean.

Moreover, if a ring $R$ is a weakly $g(x)$-nil clean, then it is weakly $g(x)$-clean. Indeed, if we let $x \in R$, then $x-1=n+s$ or $x-1=n-s$ where $n \in N(R)$ and $g(s)=0$. So, $x=(n+1)+s$ or $x=(n+1)-s$ where $n+1 \in U(R)$.

The converse is not true in general. For example, simple computations show that the ring $R=T_{2}\left(\mathbb{Z}_{3}\right)$ is (weakly) $\left(x^{4}-1\right)$-clean which is not weakly $\left(x^{4}-1\right)$-nil clean.

For any two rings $R$ and $S$, consider the ring homomorphism $\mu: C(R) \rightarrow$ $C(S)$ with $\mu\left(1_{R}\right)=1_{S}$. If $g(x)=\sum_{i=0}^{n} a_{i} x^{i} \in C(R)[x]$, we let $g_{\mu}(x):=\sum_{i=0}^{n} \mu\left(a_{i}\right) x^{i} \in$ $C(S)[x]$. In particular, if $g(x) \in \mathbb{Z}[x]$, then $g_{\mu}(x)=g(x)$.

Next, we give some properties of the class of weakly $g(x)$-nil clean rings. We start by a simple result.

Proposition 2. Let $R$ and $S$ be two rings, $\mu: R \rightarrow S$ be a ring epimorphism and $g(x)=\sum_{i=0}^{n} a_{i} x^{i} \in C(R)[x]$. If $R$ is weakly $g(x)$-nil clean, then $S$ is weakly $g_{\mu}(x)$-nil clean.

Let $R$ and $S$ be two rings such that $R$ is weakly $g(x)$-nil clean. If there is an epimorphism $\mu: R \rightarrow S$, then we say that $S$ is a weakly $g^{*}(x)$-nil clean. The verifications of the following assertions are left as exercises.

Corollary 3. Let $R$ and $S$ be ring. The following hold:

(1) If $I$ is an ideal in $R$ and $R$ is weakly $g(x)$-nil clean, then $R / I$ is weakly $g^{*}(x)$-nil clean. Moreover, the converse holds if $I$ is nil and the roots of $g^{*}(x)$ lift modulo $I$. 
(2) If the upper triangular matrix ring $T_{n}(R)$ is weakly $g(x)$-nil clean, then $R$ is weakly $g^{*}(x)$-nil clean.

(3) If the skew formal power series $R[[x, \alpha]]$ (or in particular $R[[x]]$ ) over $R$ is weakly $g(x)$-nil clean, then $R$ is weakly $g^{*}(x)$-nil clean.

(4) Let $M$ be an $(R, S)$-bimodule and $T=\left[\begin{array}{cc}A & M \\ 0 & B\end{array}\right]$ be the formal triangular matrix ring. If $T$ is weakly $g(x)$-nil clean, then $R$ and $S$ are weakly $g^{*}(x)$-nil clean.

(5) If $R$ is commutative and $M$ an $R$-module. Then the idealization $R(M)$ of $R$ and $M$ is weakly $g(x)$-nil clean if and only if $R$ is weakly $g^{*}(x)$-nil clean.

Proposition 4. Let $R_{1}, R_{2}, \ldots, R_{m}$ be rings, $R=\prod_{i=1}^{m} R_{i}$ and $g(x) \in \mathbb{Z}[x]$. Then $R$ is weakly $g(x)$-nil clean if and only if there exist $k \in\{1,2, \ldots, m\}$ such that $R_{k}$ is weakly $g(x)$-nil clean and $R_{j}$ is $g(x)$-nil clean for all $j \neq k$.

Proof. $\Rightarrow)$ : For each $i \in\{1,2, \ldots, m\}, R_{i}$ is a homomorphic image of $R$ under the projection homomorphism. Hence, $R_{i}$ is weakly $g(x)$-nil clean by Proposition 2. Without loss of generality assume that neither $R_{1}$ nor $R_{2}$ are $g(x)$-nil clean. Then there exist $r_{1} \in R_{1}$ and $r_{2} \in R_{2}$ such that $r_{1}$ is not a sum of a nilpotent and a root of $g(x)$ and $r_{2}$ is not a difference of a nilpotent and a root of $g(x)$. It follows that $\left(r_{1}, r_{2}\right)$ is not weakly $g(x)$-nil clean in $R_{1} \times R_{2}$, a contradiction.

$\Leftarrow)$ : Assume that $R_{k}$ is weakly $g(x)$-nil clean for a fixed $k \in\{1,2, \ldots, m\}$ and $R_{j}$ is $g(x)$-nil clean for all $j \neq k$. Let $r=\left(r_{i}\right) \in R$. Then there exist $n_{k} \in N\left(R_{k}\right)$ and a zero $s_{k}$ of $g(x)$ such that $r_{k}=n_{k}+s_{k}$ or $r_{k}=n_{k}-s_{k}$. If $r_{k}=n_{k}+s_{k}$, then for each $i \neq k$, write $r_{i}=n_{i}+s_{i}$ where $n_{i} \in N\left(R_{i}\right)$ and $g\left(s_{i}\right)=0$. Therefore, $r=\left(n_{i}\right)+\left(s_{i}\right)$ is a sum of a nilpotent and a zero of $g(x)$. If $r_{k}=n_{k}-s_{k}$, then for $i \neq k$, write $r_{i}=n_{i}-s_{i}$ where $n_{i} \in N\left(R_{i}\right)$ and $g\left(s_{i}\right)=0$. Therefore, $r=\left(n_{i}\right)-\left(s_{i}\right)$ is a difference of a nilpotent and a zero of $g(x)$. Therefore, $R$ is weakly $g(x)$-nil clean.

Generally, the ring of polynomials $R[t]$ is not weakly $g(x)$-nil clean ring for non-zero polynomial $g(x) \in C(R)[x]$. For example, let $R$ be any commutative ring and let $g(x)=x$. Then $t \in R[t]$ is not weakly $g(x)$-nil clean. Indeed, if $t=n \pm s$ where $n \in N(R[t])$ and $g(s)=0$, then it must be that $s=0$ and $t=n$. But $t \notin N(R[t])$, so $R[t]$ is not weakly $g(x)$-nil clean.

Let $R$ be a ring with an identity and $S$ be a ring (not necessary unitary) which is an $(R, R)$-bimodule such that $\left(s_{1} s_{2}\right) a=s_{1}\left(s_{2} a\right), a\left(s_{1} s_{2}\right)=\left(a s_{1}\right) s_{2}$ and $\left(s_{1} a\right) s_{2}=s_{1}\left(a s_{2}\right)$ for all $a \in R, s_{1}, s_{2} \in S$. The ideal-extension $I(R, S)$ of $R$ by $S$ is defined as the additive abelian group $I(R, S)=R \oplus S$ with multiplication 
$\left(a_{1}, s_{1}\right)\left(a_{2}, s_{2}\right)=\left(a_{1} a_{2}, a_{1} s_{2}+s_{1} a_{2}+s_{1} s_{2}\right)$. If $g(x)=\left(a_{0}, s_{0}\right)+\left(a_{1}, s_{1}\right) x+\ldots+$ $\left(a_{n}, s_{n}\right) x^{n} \in C(I(R, S))[x]$, then clearly $g_{R}(x)=a_{0}+a_{1} x+\ldots+a_{n} x^{n} \in C(R)[x]$.

Proposition 5. Let $R$ and $S$ as above. If $I(R, S)$ is weakly $g(x)$-nil clean, then $R$ is weakly $g_{R}(x)$-nil clean.

Proof. If we define $\mu_{R}: I(R, S) \rightarrow R$ by $\mu_{R}(r, s)=r$, then $\mu_{R}$ is a ring epimorphism. The result follows by Proposition 2 .

Let $R$ be a ring and $\alpha: R \rightarrow R$ be a ring endomorphism. By $R[[x, \alpha]]$, we denote the ring of skew formal power series over $R$; that is all formal power series in $x$ with coefficients from $R$ with multiplication defined by $x r=\alpha(r) x$ for all $r \in R$. In particular, $R[[x]]=R\left[\left[x, 1_{R}\right]\right]$ is the ring of formal power series over $R$. The skew polynomial ring $R[x, \alpha]$ can be defined in an analogous way. One can prove that $R[[x, \alpha]] \simeq I(R,\langle x\rangle)$ where $\langle x\rangle$ is the ideal generated by $x$.

Corollary 6. Let $R$ be a ring and $\alpha: R \rightarrow R$ be a ring endomorphism. If $R[[x, \alpha]]$ (or in particular $R[[x]]$ ) is weakly $g(x)$-nil clean, then $R$ is weakly $g_{\mu}(x)$-nil clean where $\mu: R[[x, \alpha]] \rightarrow R$ is defined by $\mu(f)=f(0)$.

A Morita Context $(A, B, V, W, \psi, \phi)$ consists of two rings $A, B$, two bimodules ${ }_{A} V_{B},{ }_{B} W_{A}$ and a pair of bimodule homomorphisms $\psi: V \otimes_{B} W \rightarrow A$, $\phi: W \otimes_{B} V \rightarrow B$, such that $\psi(v \otimes w) v^{\prime}=v \phi\left(w \otimes v^{\prime}\right), \phi(w \otimes v) w^{\prime}=w \psi(v \otimes$ $\left.w^{\prime}\right)$. With such a Morita context we associate the $\operatorname{ring} T=\left[\begin{array}{cc}A & V \\ W & B\end{array}\right]=$ $\left\{\left[\begin{array}{cc}a & v \\ w & b\end{array}\right] \mid a \in A, b \in B, v \in V, w \in W\right\}$ under the usual matrix addition and multiplication defined as:

$$
\left[\begin{array}{ll}
a & v \\
w & b
\end{array}\right]\left[\begin{array}{cc}
a^{\prime} & v^{\prime} \\
w^{\prime} & b^{\prime}
\end{array}\right]=\left[\begin{array}{cc}
a a^{\prime}+\psi\left(v \otimes w^{\prime}\right) & a v^{\prime}+v b^{\prime} \\
w a^{\prime}+b w^{\prime} & \phi\left(w \otimes v^{\prime}\right)+b b^{\prime}
\end{array}\right] \text {. We call } T \text { a }
$$

Morita Context ring. If $g(x)=\left[\begin{array}{cc}a_{0} & v_{1} \\ m_{0} & b_{0}\end{array}\right]+\left[\begin{array}{cc}a_{1} & v_{2} \\ m_{1} & b_{1}\end{array}\right] x+\ldots+\left[\begin{array}{cc}a_{n} & v_{n} \\ m_{n} & b_{n}\end{array}\right] x^{n} \in$ $C(T)[x]$, then clearly $g_{A}(x)=a_{0}+a_{1} x+\ldots+a_{n} x^{n} \in C(A)[x]$ and $g_{B}(x)=$ $b_{0}+b_{1} x+\ldots+b_{n} x^{n} \in C(B)[x]$.

Proposition 7. Let $T=\left[\begin{array}{cc}A & V \\ W & B\end{array}\right]$ be a Morita Context with $\psi, \phi=0$. If $T$ is weakly $g(x)$-nil clean, then $A$ is weakly $g_{A}(x)$-nil clean and $B$ is weakly $g_{B}(x)$-nil clean.

Proof. Assume that $T$ is weakly $g(x)$-nil clean with $\psi, \phi=0$, Let $I=$ $\left[\begin{array}{cc}0 & V \\ W & B\end{array}\right]$ and $J=\left[\begin{array}{cc}A & V \\ W & 0\end{array}\right]$. Then clearly $I$ and $J$ are ideals of $T$ and 
moreover, $T / I \cong A$ and $T / J \cong B$. It follows by Proposition 3 that $A$ is weakly $g_{A}(x)$-nil clean and $B$ is weakly $g_{B}(x)$-nil clean.

Corollary 8. Let $A, B$ be two rings and $M$ be an $(A, B)$-bimodule. Let $T=\left[\begin{array}{cc}A & M \\ 0 & B\end{array}\right]$ be the formal triangular matrix ring. If $T$ is weakly $g(x)$-nil clean, then $A$ is weakly $g_{A}(x)$-nil clean and $B$ is weakly $g_{B}(x)$-nil clean.

In the following Proposition, we consider a particular case of formal triangular matrix rings. Let $R$ be a commutative ring and $M$ an $R$-module. The trivial extension of $R$ by $M$ is the (commutative) ring

$$
R(M)=\left\{\left[\begin{array}{cc}
r & m \\
0 & r
\end{array}\right]: r \in R, m \in M\right\}
$$

with the usual matrix addition and multiplication. We note that if $\left[\begin{array}{cc}r & m \\ 0 & r\end{array}\right] \in$ $N(R(M))$, then clearly $r \in N(R)$. We recall that $R$ naturally embeds into $R(M)$ via $r \rightarrow\left[\begin{array}{ll}r & 0 \\ 0 & r\end{array}\right]$. Thus any polynomial $g(x)=\sum_{i=0}^{n} a_{i} x^{i} \in R[x]$ can be written as $g(x)=\sum_{i=0}^{n}\left[\begin{array}{cc}r_{i} & 0 \\ 0 & r_{i}\end{array}\right] x^{i} \in R(M)[x]$ and conversely.

Proposition 9. Let $R$ be a commutative ring and $M$ an $R$-module. Then the idealization $R(M)$ of $R$ and $M$ is weakly $g(x)$-nil clean if and only if $R$ is weakly $g(x)$-nil clean.

$$
\text { Proof. } \Rightarrow) \text { : Note that } R \simeq R(M) / \widetilde{M} \text { where } \widetilde{M}=\left\{\left[\begin{array}{cc}
0 & m \\
0 & 0
\end{array}\right]: m \in M\right\} .
$$
Hence, $R$ is weakly $g(x)$-nil clean by Proposition 2 .

$$
\Leftarrow) \text { : Let } g(x)=\sum_{i=0}^{n} a_{i} x^{i} \in R[x] \text { and } r \in R \text {. Since } R \text { is weakly } g(x) \text {-nil }
$$
clean, we have $r=n \pm s$ where $n \in N(R)$ and $g(s)=0$. Then for $m \in M$, $\left[\begin{array}{cc}r & m \\ 0 & r\end{array}\right]=\left[\begin{array}{cc}n & m \\ 0 & n\end{array}\right] \pm\left[\begin{array}{cc}s & 0 \\ 0 & s\end{array}\right]$ where $\left[\begin{array}{cc}n & m \\ 0 & n\end{array}\right] \in N(R(M))$. Moreover, we have

$$
\begin{aligned}
& g\left(\left[\begin{array}{ll}
s & 0 \\
0 & s
\end{array}\right]\right)=a_{0}\left[\begin{array}{ll}
1 & 0 \\
0 & 1
\end{array}\right]+a_{1}\left[\begin{array}{cc}
s & 0 \\
0 & s
\end{array}\right]+a_{2}\left[\begin{array}{cc}
s^{2} & 0 \\
0 & s^{2}
\end{array}\right]+\ldots+a_{n}\left[\begin{array}{cc}
s^{n} & 0 \\
0 & s^{n}
\end{array}\right] \\
= & {\left[\begin{array}{cc}
a_{0}+a_{1} s+a_{2} s^{2}+\ldots+a_{n} s^{n} & 0 \\
0 & a_{0}+a_{1} s+a_{2} s^{2}+\ldots+a_{n} s^{n}
\end{array}\right]=\left[\begin{array}{ll}
0 & 0 \\
0 & 0
\end{array}\right] . }
\end{aligned}
$$

Therefore, $R(M)$ is weakly $g(x)$-nil clean. 
If $R$ is a weakly $g(x)$-nil clean and $\theta: R \rightarrow R$ is a ring endomorphism then for any $n$, the quotient $W=R[x ; \theta] /\left\langle x^{n}\right\rangle$, where $R[x ; \theta]$ is the Hilbert twist, is weakly $g(x)$-nil clean. Indeed if $f=a_{0}+a_{1} x+a_{2} x^{2}+\ldots+a_{n-1} x^{n-1} \in W$ and $a_{0}=n \pm s$ where $n \in N(R)$ and $g(s)=0$, then $f=(f-s)+s$ or $f=(f+s)-s$ is a weakly $g(x)$-nil clean decomposition of $f$ in $W$.

\section{Weakly $x(x-b)-($ nil $)$ Clean Rings}

In this section, we consider weakly $x(x-b)$-(nil) clean rings for $b \in C(R) \cap U(R)$ and we investigate the relation between weakly $x(x-b)$-(nil) clean rings and weakly (nil) clean rings.

Theorem 10. Let $R$ be a ring and $b \in C(R)$. Then $R$ is weakly clean and $b \in U(R)$ if and only if $R$ is weakly $x(x-b)$-clean.

Proof. $\Rightarrow)$ : Suppose $R$ is weakly clean with $b \in U(R)$ and let $r \in R$. Write $r b^{-1}=u \pm e$, where $u$ is a unit and $e$ is an idempotent. Then, $r=u b \pm e b$ where clearly $u b \in U(R)$ and $e b$ is a root of $x(x-b)$. Hence, $R$ is weakly $x(x-b)$-clean.

$\Leftarrow)$ : Suppose $R$ is weakly $x(x-b)$-clean. Write $0=u \pm s$ where $u \in U(R)$ and $s(s-b)=0$. Then $s= \pm u \in U(R)$ and $s-b=0$, whence $b \in U(R)$. Now, let $r \in R$ and write $r b=u \pm s$ where $u \in U(R)$ and $s(s-b)=0$. Then, $r=u b^{-1} \pm s b^{-1}$ where $u b^{-1} \in U(R)$. Moreover,

$$
\left(s b^{-1}\right)^{2}=s(s-b+b) b^{-2}=s(s-b)+s b b^{-2}=s b^{-1}
$$

Therefore, $R$ is weakly nil clean.

In [12], the authors proved that for a ring $R$ with $b-a \in C(R), R$ is clean with $b-a \in U(R)$ if and only if $R$ is $(x-a)(x-b)$-clean. However, this equivalence can not be generalized for the weakly case. For example, the ring $R=\mathbb{Z}_{(3)} \cap \mathbb{Z}_{(5)}=\left\{\frac{a}{b}: a, b \in \mathbb{Z}, a \neq 0,3 \nmid b\right.$ and $\left.5 \nmid b\right\}$ is weakly clean, [1]. On the other hand, one can see that $R$ is not weakly $(x-7)(x-8)$-clean.

The following corollary is a direct consequence of Theorem 10 .

Corollary 11. Let $R$ be a ring and let $n \in \mathbb{N}$. The following are equivalent:

(1) $R$ is weakly clean and $2 \in U(R)$.

(2) $R$ is weakly $\left(x^{2}-2^{n} x\right)$-clean.

(3) $R$ is weakly $\left(x^{2}+2^{n} x\right)$-clean.

(4) $R$ is weakly $\left(x^{2}-2 x\right)$-clean.

(5) $R$ is weakly $\left(x^{2}+2 x\right)$-clean. 
Definition 12. Let $R$ be a ring. Then $R$ is said to have the involution property (nil involution property) if for every $r \in R$, we have $r=u+v$ where $u \in U(R)(u \in N(R)+1)$ and $v^{2}=1$. More general, for a positive integer $m$, we say that $R$ has the $m$-involution (nil $m$-involution) property if for every $r \in R$, we have $r=u+v$ where $u \in U(R)(u \in N(R)+1)$ and $v^{m}=1$.

The following characterization of the $m$-involution property is useful for the coming main theorem.

Lemma 13. Let $R$ be a ring and let $k, m \in \mathbb{N}$. Then $R$ has the $m$ involution property (nil $m$-involution property) if and only if for every $r \in R$, we have $r=u+v$ where $u \in U(R)(u \in N(R)+1))$ and $(v-k)^{m}=1$.

Proof. Suppose $R$ has the $m$-involution property. If $r \in R$, then $r-k=$ $u+w$ where $u \in U(R)$ and $w^{m}=1$. Hence, $r=u+(w+k)=u+v$ where $(v-k)^{m}=w^{m}=1$. Conversely, let $r \in R$ and write $r+k=u+w$ where $u \in U(R)$ and $(w-k)^{m}=1$. Then $r=u+(w-k)=u+v$ where $v^{m}=1$. A similar procedure can be used to prove the case of nil $m$-involution property.

It is clear that $s$ is a zero of $x^{2}-1$ if and only if $-s$ is so. Hence, weakly $\left(x^{2}-1\right)$-clean rings are the same as $\left(x^{2}-1\right)$-clean rings. Thus, according to $[12]$, we have

Corollary 14. Let $R$ be a ring. The following are equivalent:

(1) $R$ is clean and $2 \in U(R)$.

(2) $R$ is weakly $\left(x^{2}-1\right)$-clean.

(3) $R$ is $\left(x^{2}-2 x\right)$-clean.

(4) $R$ has the involution property.

Theorem 15. Let $R$ be a ring and $b \in C(R)$. Then $R$ is weakly nil clean and $b \in U(R)$ if and only if $R$ is weakly $x(x-b)$-nil clean.

Proof. Suppose $R$ is weakly nil clean and $b \in U(R)$. For $r \in R$, choose $n \in N(R)$ and $e \in I d(R)$ such that $r b^{-1}=n \pm e$. Then, $r=n b \pm e b$ where clearly $n b \in N(R)$ and $e b$ is a zero of $x(x-b)$. Therefore, $R$ is weakly $x(x-b)$-nil clean.

Conversely, suppose $R$ is weakly $x(x-b)$-nil clean. Write $1=n \pm s$ where $n \in N(R)$ and $s(s-b)=0$. Then $s= \pm(n-1) \in U(R)$ and so $b=s \in U(R)$. Now, let $r \in R$ and write $r b=n \pm s$ where $n \in N(R)$ and $s(s-b)=0$. Then, $r=n b^{-1} \pm s b^{-1}$. As in the proof Theorem 10, $s b^{-1} \in \operatorname{Id}(R)$ and clearly $n b^{-1} \in N(R)$. It follows that $R$ is weakly nil clean. 
In particular, we conclude that a ring $R$ is weakly nil clean and $2 \in U(R)$ if and only if $R$ is weakly $\left(x^{2}-2 x\right)$-nil clean.

Lemma 16. [4]. If a ring $R$ is weakly nil clean, then 6 is a (central) nilpotent element in $R$.

Since 6 is a central nilpotent in any weakly nil clean ring $R$, then $6 b$ is nilpotent for any $b \in R$. It follows that $6 b \pm 1 \in U(R)$. Hence, the following characterizations of weakly nil clean rings follow from Theorem 15

Corollary 17. Let $R$ be a ring and $b \in C(R)$. The following are equivalent

(1) $R$ is weakly nil clean.

(3) $R$ is weakly $x(x-(6 b+1))$-nil clean.

(4) $R$ is weakly $x(x-(6 b-1))$-nil clean.

For example, weakly nil clean rings are equivalent to weakly $\left(x^{2} \pm 5 x\right)$-nil clean and weakly $\left(x^{2} \pm 7 x\right)$-nil clean rings.

It is clear that if $s$ is a unit in a ring $R$ with $0=n \pm s$ for some $n \in N(R)$, then $R$ is the zero ring. Thus, we need only to consider the weakly $g(x)$ nil*clean property when the constant term of $g(x)$ is a unit. Indeed, suppose $g(x)=x^{k}+a_{k-1} x^{k-1}+\ldots+a_{1} x+a_{0} \in C(R)[x]$ with $a_{0} \in U(R)$ and $g(s)=0$. Then $s\left(s^{k-1}+a_{k-1} s^{k-2}+\ldots+a_{1}\right)=-a_{0} \in U(R)$ and so $s \in U(R)$. Examples of such polynomials are of the form $g(x)=x^{k}-1$ and $g(x)=x^{k}+1$ for $k \in \mathbb{N}$. Let $p$ be a prime integer. By the well known Fermat theorem, the field $Z_{p}$ is an examples of a weakly $\left(x^{p-1}-1\right)$-nil* clean ring which is not weakly $\left(x^{p-1}-1\right)$-nil clean. However weakly $g(x)$-nil*clean and weakly $g(x)$-nil clean coincide if all roots of $g(x)$ are non units.

Proposition 18. A ring $R$ is weakly $\left(x^{2}-1\right)$-nil* clean if and only if it has the nil involution property.

Proof. Suppose $R$ is weakly $\left(x^{2}-1\right)$-nil*clean and let $r \in R$. If $r=2$, then $r=(0+1)+1$ is a sum of a unipotent and an involution. For $r \neq 2$, we may write $r-2=n \pm s$ where $n \in N(R)$ and $s^{2}=1$. If $r-2=n+s$, then $r=(n+1)+(s+1)$ where $n+1 \in N(R)+1$ and $(s+1-1)^{2}=1$. If $r-2=n-s$, then $r=(n+1)+(1-s)$ where $(1-s-1)^{2}=1$. Thus, $R$ has the nil involution property by Lemma 13 . Conversely, if $R$ has the nil involution property and $0 \neq r \in R$, then $r+1=(n+1)+v$ where $n \in N(R)$ and $(v-1)^{2}=1$. Therefore, $r=(1+n)+(v-1)$ is the required decomposition of $r$.

Next, we generalize the equivalence in Proposition 18 to any arbitrary odd $k \in \mathbb{N}$. 
Proposition 19. Let $R$ be a ring and $k$ be an odd integer with $k \geq 3$. Consider the following statements:

(1) $R$ has the nil $(k-1)$-involution property.

(2) $R$ is weakly $\left(x^{k-1}-1\right)$-nil* clean.

(3) $R$ is weakly $\left(x^{k}-x\right)$-nil clean.

Then $(1) \Leftrightarrow(2)$ and $(2) \Rightarrow(3)$. If moreover, aR (or $R a$ ) contains no non trivial idempotents for every $a \in R$, then the three statements are equivalent.

Proof. $(1) \Leftrightarrow(2)$ : Suppose $R$ has the nil $(k-1)$-involution property and let $0 \neq r \in R$. Write $r+1=u+v$ where $u \in N(R)+1$ and $v^{k-1}=1$. Then $r=(u-1)+v$ where $u-1 \in N(R)$ and $v$ is a root of $x^{k-1}-1$. Conversely, suppose $R$ is weakly $\left(x^{k-1}-1\right)$-nil* clean. Let $r \in R$. If $r=2$, then $r=(0+1)+1$. For $r \neq 2$, we write $r-2=n \pm s$ for $n \in N(R)$ and $s^{k-1}=1$. If $r-2=n+s$, then $r=(n+1)+(s+1)$ where $n+1 \in N(R)+1$ and $(s+1-1)^{k-1}=1$. If $r-2=n-s$, then $r=(n+1)+(1-s)$ where $(1-s-1)^{k-1}=1$ since $k-1$ is even. In both cases, $R$ has the nil $(k-1)$-involution property by Lemma 13 .

$(2) \Rightarrow(3)$ : The proof is obvious.

Now, we assume that for every $a \in R, a R$ (or $R a$ ) contains no non trivial idempotents and prove $(3) \Rightarrow(1)$.

$(3) \Rightarrow(1)$ : suppose $R$ is weakly $\left(x^{k}-x\right)$-nil clean. Let $a \in R$ and write $a-1=n \pm s$ where $n \in N(R)$ and $s^{k}=s$. Then $a s^{k-1}=(n+1) s^{k-1} \pm s$ and so $a\left(1-s^{k-1}\right)=(n+1)\left(1-s^{k-1}\right)=u\left(1-s^{k-1}\right)$ where $u \in U(R)$. Since clearly $u\left(1-s^{k-1}\right) u^{-1}=a\left(1-s^{k-1}\right) u^{-1} \in a R$ is an idempotent, then by assumption $u\left(1-s^{k-1}\right) u^{-1}=0$. Therefore $s^{k-1}=1$ and $a=(n+1)+( \pm s)$ where $( \pm s)^{k-1}=1$ again since $k-1$ is even. The case when $R a$ contains no non trivial idempotent for every $a \in R$ is similar. Therefor, $R$ has the nil $(k-1)$-involution property.

It is easy to see that the ring $\mathbb{Z}_{4}$ is a (weakly) $\left(x^{4}-x\right)$-nil clean ring with $a \mathbb{Z}_{4}$ contains no non trivial idempotent for every $a \in \mathbb{Z}_{4}$. But, $\mathbb{Z}_{4}$ is not weakly $\left(x^{3}-1\right)$-nil*clean. Therefore, the equivalence of the three statements in Proposition 19 does not hold for an even integer $k$.

Recall that for a ring $R$ and $n \in \mathbb{N}$, we denote by $U_{n}(R)$ the set of elements in $R$ that are either units or sum of $n$ units. We now generalize the implication $(2) \Rightarrow(3)$ in the previous theorem to more general $g(x)$.

Proposition 20. Let $R$ be a ring, $k \in \mathbb{N}$ and $g(x)=x^{k}+a_{k-1} x^{k-1}+$ $\ldots+a_{1} x+a_{0} \in C(R)[x]$ where $a_{0} \in U(R)$. If $R$ is weakly $g(x)$-nil ${ }^{*}$ clean, then $R=U_{2}(R)$. In particular, if $R$ is weakly $\left(x^{k-2}+x^{k-3}+\ldots+x+1\right)$-nil* clean, then $R=U_{2}(R)$ is weakly $\left(x^{k}-x\right)$-nil clean. 
Proof. Suppose $R$ is weakly $g(x)$-nil* clean and let $r \in R$. If $r=1$, then $r \in U_{2}(R)$. Suppose $r \neq 1$ and let $r-1=n \pm s$ where $n \in N(R)$ and $s^{k}+a_{k-1} s^{k-1}+\ldots+a_{1} s+a_{0}=0$. Then $r=(n+1) \pm s$ where $n+1 \in U(R)$ and $s\left(s^{k-1}+a_{k-1} s^{k-2}+\ldots+a_{1}\right)=-a_{0} \in U(R)$. Therefore, $r=(n+1)+( \pm s)$ is a sum of two units in $R$ and $R=U_{n}(R)$. where $s \in U(R)$ and so $s \in U(R)$. Thus, $r \in U_{2}(R)$. Since also $1 \in U_{2}(R)$, then $R=U_{2}(R)$. In particular if $R$ is weakly $\left(x^{k-2}+x^{k-3}+\ldots+x+1\right)$-nil*clean, then $R=U_{2}(R)$ by taking $a_{0}=1$. Now, let $r \in R$. If $r=0$, then $r$ is clearly a weakly $\left(x^{k}-x\right)$-nil clean element. Let $0 \neq r \in R$ and write $r=n \pm s$ where $n \in N(R)$ and $s^{k-2}+s^{k-3}+\ldots+s+1=0$. Then $s^{k}-s=s(s-1)\left(s^{k-2}+s^{k-3}+\ldots+s+1\right)=0$ and so $R$ is weakly $\left(x^{k}-x\right)$-nil clean.

In the next result we analogue Theorem 3.1 in [2]. The proof is almost similar.

Proposition 21. Let $R$ be a ring, $k$ be an even positive integer and $a, b \in R$. Then $R$ is weakly $\left(a x^{k}-b x\right)$-nil clean if and only if $R$ is weakly $\left(a x^{k}+b x\right)$-nil clean.

However, the equivalence in Proposition 21 does not hold for odd powers. For example, The ring $\mathbb{Z}_{3}$ is clearly a (weakly) $\left(x^{3}-x\right)$-nil clean which is not weakly $\left(x^{3}+x\right)$-nil clean.

\section{References}

[1] M. S. Ahn, D. D. Anderson, Weakly clean rings and almost clean rings, Rocky Mountain J. Math. 36 (2006), 783-798.

[2] N. Ashrafi, Z. Ahmadi, Weakly $g(x)$-clean rings, Iranian Journal of Mathematical Sciences and Informatics 7 (2) (2012), 83-91.

[3] S. Breaz, G. Călugăreanu, P. Danchev and T. Micu, Nil-clean matrix rings, Linear Algebra and Appl., 439 (10)(2013), 3115-3119.

[4] S. Breaz, P. Danchev, Y. Zhou, Rings in which every element is either a sum or a difference of a nilpotent and an idempotent, preprint arXiv:1412.5544 [math.RA].

[5] V. P. Camillo, J. J. Simón, The Nicholson-Varadarajan theorem on clean linear transformations, Glasgo Math. J. 44 (2002), 365-369.

[6] M. Chacron, On a theorem of Herstein, Cand. J. Math., 21 (1969), 1348-1353.

[7] H. Chen, Strongly nil clean matrices over $R[x] /\left(x^{2}-1\right)$, Bull. Korean Math. Soc, 49 (3) (2012), 589-599.

[8] H. Chen, On Strongly Nil Clean Matrices, Comm. Algebra, 41 (3)(2013), 1074-1086.

[9] P.V. Danchev, W.Wm. McGovern, Commutative weakly nil clean unital rings, J. Algebra 425 (2015), 410-422. 
[10] A. J. Diesl, Classes of Strongly Clean Rings, Ph.D. Thesis, University of California, Berkeley, 2006.

[11] A. J. Diesl, Nil clean rings, J. Algebra, 383 (2013), 197-211.

[12] L. Fan, X. Yang, On rings whose elements are the sum of a unit and a root of a fixed polynomial, Comm. Algebra, 36 (2008), 269-278.

[13] H. A. Khashan, A. H. Handam, $g(x)$-nil clean rings, Scientiae Mathematicae Japonicae, 2 (2016), 145-154.

[14] D. Mosic, Characterizations of k-potent elements in rings, Annali di Matematica, 194 (2015), 1157-1168.

[15] W. K. Nicholson, Lifting idempotents and exchange rings, Trans. Amer. Math. Soc., 229 (1977), 269-278.

[16] W. K. Nicholson, Strongly clean rings and Fitting's lemma, Comm. Algebra, 27 (1999), 3583-3592.

[17] W. K. Nicholson, Y. Zhou, Endomorphisms that are the sum of a unit and a root of a fixed polynomial, Canad. J. Math. Bull. 49 (2006), 265-269.

[18] M. Ôhori, On strongly $\pi$-regular rings and periodic rings, Math. J. Okayama Univ., 27 (1) (1985), 49-52.

[19] S. Sahebi, M. Jahandar, A note on uniquely (nil) clean ring, Journal of Linear and Topological Algebra, 1 (2) (2012), 65-67. 